\title{
STATUS OF THE ORGANIZED RECYCLING OF CADMIUM-BEARING SECONDARY RAW MATERIALS
}

\author{
A. R. Barashev, S. V. Karelov, \\ S. V. Mamyachenkov, and O. S. Anisimova
}

UDC 621.355 .8

The main methods being used in metallurgy to systematically recycle cadmium-bearing secondary raw materials are examined. Hydrometallurgical methods which use sequestering reagents that can be regenerated are the most promising technologies from the standpoint of suitability for industrial use, environmental safety, and the selectivity with which cadmium is extracted.

Keywords: cadmium-bearing secondary raw materials, regenerable sequestering reagents.

Alkaline nickel-cadmium power sources are widely used in different areas of the economy (from mine lamps to the pulling accumulators of electric locomotives and submarines). Their favorable combination of technical-economic and electrical characteristics has made them one of the main chemical sources of electric current.

Nickel-cadmium power supplies account for about $10 \%$ of all chemical current sources in use [1]. Their volume of use and recycling in Russia cannot be estimated because there is no legal framework that regulates recycling of the hazardous wastes. Having such laws would make it possible to control the collection and recycling of storage batteries that have exhausted their service life.

Spent nickel-cadmium current sources are complex multicomponent raw materials: the phase composition and dimensions of the batteries vary widely and depend on the battery's specific function. The battery may contain different additives that improve its service properties, and the quantity and composition of these additives also vary. As a result, it is necessary to use unconventional multistage methods to recycle used batteries [2].

The organized recycling of alkaline nickel-cadmium batteries has become important for environmental, economic, and technological reasons. The selective recovery of metals from such a multicomponent resource - a resource which is characterized by a wide range of compositions - is a challenging problem that requires new research. The recycling of wastes of this type is particularly important in the case of nickel-cadmium batteries because of the simultaneous presence of substantial quantities of valuable nickel and highly toxic cadmium. Any recycling technology that is developed should therefore be designed so as to fully recover these metals in the finished product, thus preventing the formation of gaseous emissions and keeping these metals out of waste water.

Consequently, the following requirements must be met by a technology used to recycle nickel-cadmium batteries:

- it must be environmentally clean and safe;

- a high percentage of the valuable components should be recovered;

- the equipment used should be as simple as possible; and

- the technology should yield high-quality products suitable for the production of new storage batteries.

Existing pyrometallurgical methods essentially involve the sublimation of cadmium oxide at high temperatures. There are three main pyrometallurgical methods of recycling nickel-cadmium batteries:

1) sublimation of cadmium oxide in an open furnace followed by its condensation in the form of a powder [3, 4];

Yeltsin Ural Federal University (UrFU), Ekaterinburg, Russia; e-mail: a.r.barashev@ ustu.ru. Translated from Metallurg, No. 3, pp. 95-97, March, 2013. Original article submitted October 29, 2012. 
2) distillation in the atmosphere of a closed furnace with the production of powdered metallic cadmium and an ironnickel alloy [5]; and

3) chlorination of batteries by gaseous chlorine or hydrochloric acid, with cadmium chloride undergoing sublimation at $960^{\circ} \mathrm{C}$. Nickel and cobalt remain stable [6].

The processes Snam (France), Sab Nife (Sweden), and Inmetco (U.S.) are three of the large-scale pyrometallurgical technologies that have been developed for recycling nickel-cadmium batteries by distilling cadmium in the atmosphere of a closed furnace. The distillation is carried out within the temperature range $850-900^{\circ} \mathrm{C}$ in the first two processes and the resulting cadmium is more than $99.95 \%$ pure. The iron-nickel residue is remelted in electric furnaces. The production of cadmium oxide by these technologies has been repeatedly been suspended due to the extremely hazardous working conditions. The Inmetco Company in the U.S. (an affiliate of INCO) remains the only firm that recycles nickel-cadmium batteries with the use of high-temperature processes. The iron-nickel alloy obtained from these processes is used in the production of stainless steel. The cadmium is converted to gaseous form (together with zinc and lead) and dispatched for separate processing.

The recycling of cadmium-bearing raw materials by pyrometallurgical technologies has its advantages and disadvantages [7]. Among the advantages are the high unit productivity and the relatively low cost of the reagents that are used. The disadvantages are: the low quality of the products that are obtained; the need to clean the process gases and render them harmless; the small yield of valuable components, including rare metals; the need to use a hermetic vessel and a complicated dust-catching system in connection with the high toxicity of cadmium and its compounds.

In most cases, the products obtained from recycling wastes in pyrometallurgical units require additional (often hydrometallurgical) processing, which makes pyrometallurgical methods significantly less cost-effective. Also, it would be inadvisable to return the wastes to the stockhouse because harmful impurities would then accumulate in the main production cycle and valuable components in the raw materials would be lost with the products that are disposed of.

Another shortcoming of pyrometallurgical methods is the large consumption of fuel and energy, since the working temperatures are within the range $800-1000^{\circ} \mathrm{C}$. It is difficult to compare the energy costs with the corresponding costs for hydrometallurgical processes because the latter often use electrolysis to reduce the metal.

The hydrometallurgical technologies that can be used for recycling mainly entail converting metals to a solution by basic or acid leaching. The metals can be recovered from the solution by precipitation-based methods (with a change in the $\mathrm{pH}$ of the medium), electrolysis, or liquid extraction.

The sulfuric-acid method has become the most widely used technology. In the first stage of this process, nearly all of the components of the scrap are dissolved. Different techniques are then used for separation and recovery of the metals from the solution. For example, metals are separated by the sorption of cadmium on an anion-exchange resin which contains functional groups that include nitrogen.

Ammonia leaching is used in addition to acid leaching. In order to dissolve cadmium, it is necessary to keep the concentration of $\mathrm{NH}_{3}$ at the level 4 moles $/ \mathrm{dm}^{3}$. To precipitate cadmium, the concentration of $\mathrm{NH}_{3}$ is reduced as a result of its volatilization during heating. The shortcomings of the method: significant energy use; losses of $\mathrm{NH}_{3}$; the fact that the spent solution cannot be recycled. The degree of recovery of metals from scrap is greater than $98 \%$ when this leaching method is used.

Hydrochloric acid leaching of used nickel-cadmium batteries is accompanied by the dissolution of all of the components of the scrap. Thus, the most important issue becomes selective separation of the metals to obtain monosolutions or high-quality solid products.

In recent years, there have been reports on the use of electrochemical methods to extract valuable components from used alkaline storage batteries. It should be pointed out that these methods are still in the laboratory testing stage.

The selective extraction of cadmium from model solutions obtained by leaching used $\mathrm{Ni}-\mathrm{Cd}$ batteries in inorganic acids has shown that nitric-acid-based media are not suited for recovering cadmium because nitrate ions are reduced at the potential at which cadmium is precipitated. Cathodic reduction is best carried out from media which contain chloride or sulfate ions, since in this case cadmium's precipitation voltage is $200 \mathrm{mV}$ less negative than the corresponding parameter for $\mathrm{Ni}^{2+}$. Under optimum conditions, over $90 \%$ of the cadmium has been recovered, no co-precipitation of nickel has occurred, and current efficiency has exceeded $80 \%$. 


\section{Conclusions}

1. Studies conducted to find a new technology for the systematic recycling of used alkaline nickel-cadmium batteries are important for the following reasons:

- the production of chemical current sources that contain cadmium is still being carried out on a large scale, which makes recycling the cadmium important from both an economic standpoint (providing the necessary raw materials to factories in the battery industry) and the environmental perspective;

- the tightening of environmental laws on cadmium-bearing wastes in many countries (and in Russia in the future) will soon make it mandatory to save, store, and systematically recycle used nickel-cadmium batteries.

2. A brief analysis of the chemical and phase compositions of used alkaline batteries showed that the cadmium in them is present mainly in the form of an oxide; appreciable amounts of metallic cadmium may also be present (if the scrap contains batteries that have not been discharged). The main impurities are di- and trivalent iron oxide and metallic iron (lowcarbon steel); for high-quality batteries used in aviation and shipping typically contain up to $0.6-0.9 \% \mathrm{CO}$. Small amounts of nickel oxides and hydroxides may be found in batteries that are not disassembled properly.

3. Pyrometallurgical methods of recycling used batteries have serious drawbacks despite their high unit productivity and the relatively low cost of the reagents that are used.

4. Sulfuric acid leaching of the cadmium-bearing contacts of alkaline batteries entails the dissolution of substantial quantities of iron, and precipitating iron from sulfate solutions is problematic. The selective removal of other impurities requires the use of complicated processes (usually extraction-based) involving multiple stages. The same shortcomings are intrinsic to ammonia leaching and to technologies that use nitric or hydrochloric acid.

5. From the viewpoint of environmental safety and suitability for industrial conditions, the best approach is to use hydrometallurgical methods which use sequestering reagents that can be regenerated. It have the following advantages:

- they allow the use of a simple technology to obtain quality cadmium-bearing products (an oxide and a hydroxide) which can in turn be used to make new storage batteries and to obtain an iron concentrate that can be employed in steel production;

- the relatively expensive sequestering reagent that is used can be almost completely regenerated;

- the production cycle is closed in terms of the toxic solutions that are formed, and no cadmium compounds are released into the environment;

- it is possible to use standard hydrometallurgical equipment and readily available auxiliary materials, which keeps production costs low.

This study was conducted with financial support that Ural Federal University gives young scientists as part of its development program.

\section{REFERENCES}

1. K. Fricke and N. Knudsen, Disposal of Portable Batteries, GRS Batterien, May, 2002.

2. J. Frenay and S. Feron, "Domestic battery recycling in Western Europe," Proc. of the 2nd Int. Symp. in Recycling of Metals and Engineered Materials, The Minerals, Metals, and Materials Society (1990), Vol. 2. pp. 639-647.

3. R. J. DeLisle, H. E. Martin, and A Wilkerson, U.S. Patent No. 5437705, Device and Process for the Recovery of Cadmium and Nickel, Eveready Battery Company, St. Louis, MO, filed 09.04.1994, issued 01.08.1995.

4. A. L. Melin and V. H. Svensson, U. S. Pat. No. 4401463, Process for the Recovery of Metals from the Scrap from Nickel-Cadmium Electric Storage Batteries, SAB Nife AB, Sweden, filed 09.21.1982, issued 08.30.1983.

5. A Cox and D. J. Fray, "Recycling of cadmium from domestic sealed NiCd battery waste by use of chlorination," Trans. Inst. Min. Metall., Sect. C: Miner. Process. Extr. Metall, 108, 153-158 (1999).

6. J. David, "Nickel-cadmium battery recycling evolution in Europe," Power Sources, No. 57, 71-73 (1995).

7. Huang Kui, Li Jia, and Xu Zhenming, Characterization and Recycling of Cadmium from Waste Nickel-Cadmium Batteries, School of Environmental Science and Engineering, Shanghai Jiao Tong University, PRC (2010). 